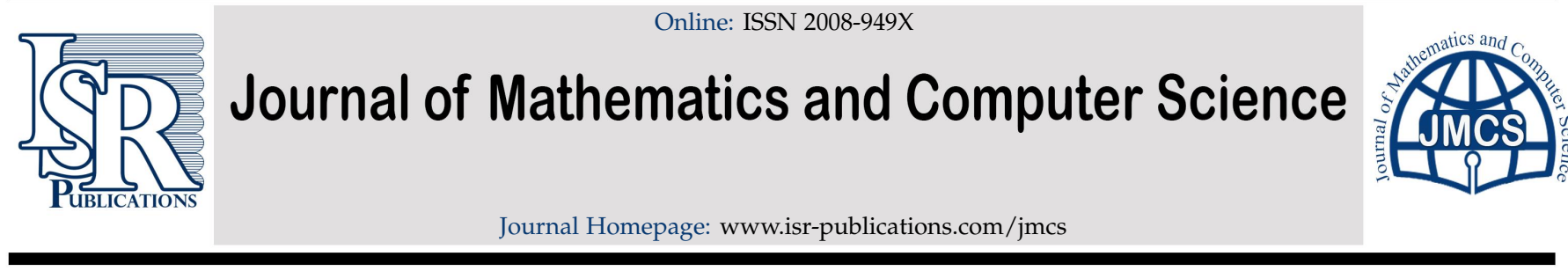

\title{
Taylor's expansion for fractional matrix functions: theory and applications
}

\author{
Ahmad El-Ajou \\ Department of Mathematics, Faculty of Science, Al Balqa Applied University, Salt 19117, Jordan. \\ Department of Mathematics, Faculty of Science, Taibah University, Madina, KSA.
}

\begin{abstract}
In this paper, several aims and tasks have been accomplished that can be summarized in the following points. Firstly, we recover some nice results related to the convergence and radii of convergence for the matrix fractional power series formula. Secondly, the Frobenius norm approximations for the matrix fractional derivatives in Caputo sense and fractional integrals in Riemann-Liouville sense are presented. Thirdly, we present the general exact and numerical solutions of four important and interesting matrix fractional differential equations and a new computational technique is also applied for getting the general solutions of the non-linear case in Caputo sense. Finally, some illustrated examples and special cases are also given and considered to show our new approach.
\end{abstract}

Keywords: Caputo fractional derivative, Riemann-Liouville integral, matrix Mittag-Leffler functions, matrix fractional differential equations.

2020 MSC: 26A33, 32A05, 15A16.

(C)2020 All rights reserved.

\section{Introduction}

The use of matrix differential equations (MDEs) are appeared in many applications and real life problems such as in matrix theory, control theory, physics phenomena, engineering problems, decay-growth problems, mortgage problems, modeling of signal processing, modeling of best predictions, simulationreduction problems, matrix time-varying descriptor systems, computing system and large scale benchmark problems, state-space problem and constrained least-squares problems [1-4, 10, 19, 23, 24, 27, 30, 39]. The general form of the first order non-homogeneous linear MDE with appropriate orders of matrices is formulated by $[1,19,23,24,30]$ :

$$
X^{\prime}(t)=\sum_{i=1}^{s} A_{i} X(t) B_{i}+G(t), X\left(t_{0}\right)=E .
$$

In addition, many interesting and important cases can be formulated from Eq. (1.1) and one of the

Email address: ajou44@bau.edu.jo, ajou42@yahoo.com (Ahmad El-Ajou)

doi: $10.22436 /$ jmcs.021.01.01

Received: 2019-08-07 Revised: 2020-01-23 Accepted: 2020-02-04 
simplest and well-known cases from Eq. (1.1) is the following $\operatorname{MDE}[1,3,19,24,30]$ :

$$
X^{\prime}(t)=A X(t)+G(t), X(0)=B,
$$

and the general exact solution of Eq. (1.2) is presented by:

$$
X(t)=e^{A t} B+\int_{0}^{t} e^{A(t-s)} G(s) d s,
$$

where $e^{A t} \in M_{m}$ is the matrix exponential function $\left(M_{m}\right.$ and $M_{m, n}$ stand for the set of all matrices of order $m \times m$ and $m \times n$, respectively). Due to the introduction of fractional calculus, scientists nowadays are paying attentions to the topic of fractional operators and its applications, since they found this topic is more fitting for extending and generalizing many classical differential equations (systems) and also many phenomena in physics, engineering and problems in control theory can be modeled mathematically by matrix fractional differential equations (MFDEs) and systems such as fractional L system, fractional Chen systems, fractional Lorenz system, descriptor and dynamic systems, nonlinear oscillation of earthquake, population of fractional oscillators and electromagnetic wave $[4,7-9,11-13,20,22,25,28,29,31,32,34-$ $38,40]$.

There are many definitions of fractional integral and fractional derivative $[9,16,17,21,31,34,35$, 37], the most important of which are the definition of Riemann-Liouville fractional integral and Caputo fractional derivative, which are defined as follows.

- The Riemann-Liouville fractional integral of order $\alpha \geqslant 0$ of $\varphi(t)$ is defined by:

$$
J_{s}^{\alpha} \varphi(t)= \begin{cases}\frac{1}{\Gamma(\alpha)} \int_{s}^{t}(t-x)^{\alpha-1} \varphi(x) d t, & t>x>s \geqslant 0, \alpha>0, \\ \varphi(t), & \alpha=0 .\end{cases}
$$

- The Caputo fractional derivative of order $\alpha>0$ of $\varphi$ is defined by:

$$
D_{s}^{\alpha} \varphi(t)= \begin{cases}J_{s}^{n-\alpha} \varphi^{(n)}(t), & t>s \geqslant 0, n-1<\alpha<n, \\ \frac{d^{n} \varphi(t)}{d t^{n}}, & \alpha=n .\end{cases}
$$

Many of the properties of the previous definitions exist in the references [6, 9, 14, 15, 18, 31, 33-35, 37]. The most important of these properties that we will need during this work can be summarized in the following lemma.

Lemma 1.1. For $\varphi(t), t \geqslant s, \alpha, \beta \geqslant 0, C \in \mathbb{R}$, and $\gamma \geqslant-1$, we have:

1. $J_{s}^{\alpha} J_{s}^{\beta} \varphi(t)=J_{s}^{\alpha+\beta} \varphi(t)=J_{s}^{\beta} J_{s}^{\alpha} \varphi(t)$,

2. $J_{s}^{\alpha} C=\frac{C}{\Gamma(\alpha+1)}(t-s)^{\alpha}$,

3. $J_{s}^{\alpha}(t-s)^{\gamma}=\frac{\Gamma(\gamma+1)}{\Gamma(\alpha+\gamma+1)}(t-s)^{\alpha+\gamma}$,

4. $\mathrm{D}_{\mathrm{s}}^{\alpha} \mathrm{C}=0$,

5. $\mathrm{D}_{\mathrm{s}}^{\alpha}(\mathrm{t}-\mathrm{s})^{\gamma}=\frac{\Gamma(\gamma+1)}{\Gamma(\gamma+1-\alpha)}(\mathrm{t}-\mathrm{s})^{\gamma-\alpha}$,

6. $D_{s}^{\alpha} J_{s}^{\alpha} \varphi(t)=\varphi(t)$,

7. $\int_{s}^{\alpha} D_{s}^{\alpha} \varphi(t)=\varphi(t)-\sum_{j=0}^{n-1} \varphi\left(s^{+}\right) \frac{(t-s)^{j}}{j !}, n-1<\alpha \leqslant n$.

Recently, Al-Zhour [2] used the Kronecer product method for solving the following non-homogeneous MFDEs in Caputo sense:

$$
D^{\alpha} X(t)=A X(t)+G(t), X\left(t_{0}\right)=B, 0<\alpha \leqslant 1,
$$

and it has the following solution:

$$
X(t)=E_{\alpha}\left(A t^{\alpha}\right) B+\int_{0}^{t}(t-s)^{\alpha-1} E_{\alpha}\left(A(t-s)^{\alpha}\right) G(s) d s,
$$

where $E_{\alpha}\left(A t^{\alpha}\right), A \in M_{m}$ is the one-parameter Matrix Mittag-Leffler function (MM-LF) and given by the 
following formula:

$$
E_{\alpha}\left(A t^{\alpha}\right)=\sum_{k=0}^{\infty} \frac{A^{k} t^{\alpha k}}{\Gamma(k \alpha+1)},
$$

whereas the two-parameter MM-LF is given by the following formula $[2,6-8,32]$ :

$$
E_{\alpha, \beta}\left(A t^{\alpha}\right)=\sum_{k=0}^{\infty} \frac{A^{k} t^{\alpha k}}{\Gamma(k \alpha+\beta)} .
$$

In a case, if we set $\alpha=1$ in Eq. (1.3), then we have:

$$
E_{1}(A t)=\sum_{k=0}^{\infty} \frac{A^{k} t^{k}}{\Gamma(k+1)}=e^{A t} .
$$

Very recently, Al-Zhour [4], Al-Zuhiri et.al. [5], and Kilicman and Ahmood [26] used, respectively, the Kronecker product method, Hadamard product method and fractional Laplace transform method for solving very restricted matrix fractional differential equations.

In this paper, we find the exact and numerical solutions of some interesting and attractive linear and non-linear MFDEs in Caputo sense by using matrix fractional power series (MFPS) method and establish some new results related to the convergence of this method. Moreover, the Frobenius norm (which is defined below) approximation for the matrix fractional derivatives in Caputo sense and fractional integrals in Riemann-Liouville sense of a given function are presented with some illustrated numerical examples to show our new approach. Note that the results shown in this paper are established in Caputo fractional derivative $D_{s}^{\alpha}$, since it has suitable for modeling MFDEs and FDEs.

The outcome of this paper is organized as follows. In the next section, we extend the generalized fractional Taylor's series to the matrix form and establish some nice results related to the convergence of the extension formula. In Section 3, we give two applications for computing the Frobenius norm approximation for the matrix fractional derivatives and fractional integrals of a given matrix function. Section 4 deals with four applications related to the linear and nonlinear MFDEs with some illustrated numerical computations in order to show the capability and simplicity of our new approach on the field of matrix theory. The conclusion of the present work is given as in the last section.

\section{Matrix fractional power series (MFPS)}

This section extends the fractional power series that discussed in the references $[14,16-18]$ to the matrix case, as general, and establish some new nice results related to the convergent and radii of convergence for MFPS in Caputo sense. In addition, we study some important definitions and theorems which are very useful to investigate our results in the approximation of the matrix fractional derivative in Caputo sense and fractional integral in Riemann-Liouville sense and also in the solutions of some MFDEs as in Sections 3 and 4.

Definition 2.1. Let $A_{k} \in M_{m, n}(k=0,1,2, \ldots)$. Then the sequence $\left\{A_{k}\right\}_{k=0}^{\infty}$ converges to a matrix $A \in$ $M_{m, n}$ with respect to a matrix norm $\|\cdot\|$ on $M_{m, n}$ if and only if $\lim _{k \rightarrow \infty}\left\|A_{k}-A\right\|=0$. If $\left\{A_{k}\right\}$ converges to $A$ with respect to $\|\cdot\|$, we write $\lim _{k \rightarrow \infty} A_{k}=A$.

Definition 2.2. Given a matrix series $\sum_{j=0}^{\infty} A_{j}$ such that $A_{j} \in M_{m, n}$ and $S_{k}$ denotes its kth partial sum, $S_{k}=\sum_{j=0}^{k} A_{j}$. If $\left\{S_{k}\right\}$ is convergent and $\lim _{k \rightarrow \infty} S_{k}=S$ is existing, then $\sum_{j=0}^{\infty} A_{j}$ is convergent to the matrix $S$. Otherwise, the series is divergent.

Theorem 2.3. Let $A_{k} \in M_{m, n}(k=0,1,2, \ldots)$ and the matrix series $\sum_{k=0}^{\infty} A_{k}$ be convergent, then $\lim _{k \rightarrow \infty} A_{k}=$ 0. 
Proof. Since $\sum_{k=0}^{\infty} A_{k}$ is convergent, then $\left\{S_{k}\right\}$ is convergent and also since $\lim _{k \rightarrow \infty} S_{k}=S$, then $\lim _{k \rightarrow \infty} S_{k-1}=S$. Therefore, $\lim _{k \rightarrow \infty} A_{k}=\lim _{k \rightarrow \infty}\left(S_{k}-S_{k-1}\right)=0$.

Definition 2.4. Let $A_{k} \in M_{m, n}(k=0,1,2, \ldots)$ be a sequence of constant matrices, $0 \leqslant r-1<\alpha \leqslant r$ and the variable $t \geqslant t_{0}$, then the following series

$$
\sum_{k=0}^{\infty} A_{k}\left(t-t_{0}\right)^{k \alpha},
$$

is called the MFPS about $t_{0}$ and $A_{k}(k=0,1,2, \ldots)$ are called the coefficients of the MFPS.

Remark 2.5. The MFPS as in Eq. (2.1) always converges when $t=t_{0}$.

Remark 2.6. We shall treat the MFPS as in Eq. (2.1) about $t_{0}=0$ since the translation $t^{\prime}=t-t_{0}$ reduces the MFPS about $t_{0}$ to the case about 0 .

Theorem 2.7. Let $A_{k} \in M_{m, n}(k=0,1,2, \ldots)$. Then for $\mathrm{t} \geqslant 0$, we have:

(i) if the MFPS $\sum_{k=0}^{\infty} A_{k} t^{k \alpha}$ converges when $\mathrm{t}=\lambda>0$ with respect to a matrix norm $\|\cdot\|$, then it also converges when $0 \leqslant \mathrm{t}<\lambda$;

(ii) if the MFPS $\sum_{k=0}^{\infty} A_{k} t^{k \alpha}$ diverges when $t=\rho>0$, then it also diverges when $t>\rho$.

Proof.

(i). Assume that $\sum A_{k} \lambda^{k \alpha}$ converges, then $\lim _{k \rightarrow \infty} A_{k} \lambda^{k \alpha}=0$ (by Theorem 2.3). That is $\exists$ a positive number $N$ and a matrix norm $\|\cdot\|$ on $M_{m, n}$ such that $\left\|A_{k} \lambda^{k \alpha}\right\|<\varepsilon=1$ when $k \geqslant N$. Thus, for $k \geqslant N$, we have:

$$
\left\|A_{k} \lambda^{k \alpha}\right\|=\left\|\frac{A_{k} \lambda^{k \alpha} t^{k \alpha}}{\lambda^{k \alpha}}\right\|=\left\|A_{k} \lambda^{k \alpha}\right\|\left|\frac{t}{\lambda}\right|^{k \alpha}<\left|\frac{t}{\lambda}\right|^{k \alpha} .
$$

Now, if $0 \leqslant t<\lambda$, then $\left|\frac{t}{\lambda}\right|^{\alpha}<1$ and $\sum\left|\frac{t}{\lambda}\right|^{k \alpha}$ is convergent and so $\sum_{k=0}^{\infty}\left\|A_{k} t^{k \alpha}\right\|$ is a convergent series (by comparison test) which implies that $\sum A_{k} t^{k \alpha}$ is convergent.

(ii). Part (ii) follows by applying Part (i) of Theorem 2.7.

Theorem 2.8. The MFPS $\sum_{\mathrm{k}=0}^{\infty} A_{\mathrm{k}} \mathrm{t}^{\mathrm{k} \alpha}$ has the following three cases:

(i) converges at $\mathrm{t}=0$;

(ii) converges for each $t \geqslant 0$;

(iii) converges when $0 \leqslant \mathrm{t}<\mathrm{R}$ and diverges when $\mathrm{t}>\mathrm{R}$, where $\mathrm{R}$ is a positive integer number and called the "radius of convergence" of the MFPS.

Proof. Follows by the same techniques of the proof as in [14, Theorem 3.2].

Remark 2.9. It is clear that $R=0$ in case (i) and $R=\infty$ in case (ii) of Theorem 2.8.

Theorem 2.10. The matrix classical power series (MCPS) $\sum_{k=0}^{\infty} A_{k} t^{k},-\infty<t<\infty$ has a radius of convergence $R$ if and only if the MFPS $\sum_{k=0}^{\infty} A_{k} t^{k \alpha}, t \geqslant 0$ has radius of convergence $R^{1 / \alpha}$.

Proof. Straightforward by changing the variable $t=x^{\alpha}, x \geqslant 0$ and conversely by changing the variable $t=x^{1 / \alpha}, x \geqslant 0$.

Theorem 2.11. If the MFPS $\sum_{k=0}^{\infty} A_{k} t^{k \alpha}, t \geqslant 0$ has radius of convergence $R>0$ and $X(t)=\sum_{k=0}^{\infty} A_{k} t^{k \alpha} \in$ $M_{m, n}, 0 \leqslant t<R, 0 \leqslant r-1<\alpha \leqslant r$, then we have

$$
\begin{aligned}
& \mathrm{D}_{0}^{\alpha} \mathrm{X}(\mathrm{t})=\sum_{\mathrm{k}=1}^{\infty} A_{\mathrm{k}} \frac{\Gamma(\mathrm{k} \alpha+1)}{\Gamma((\mathrm{k}-1) \alpha+1)} \mathrm{t}^{(\mathrm{k}-1) \alpha,} \\
& \mathrm{J}_{0}^{\alpha} \mathrm{X}(\mathrm{t})=\sum_{\mathrm{k}=0}^{\infty} A_{\mathrm{k}} \frac{\Gamma(\mathrm{k} \alpha+1)}{\Gamma((\mathrm{k}+1) \alpha+1)} \mathrm{t}^{(\mathrm{k}+1) \alpha} .
\end{aligned}
$$


Proof. Define $Y(x)=\sum_{k=0}^{\infty} A_{k} x^{k}$ for $0 \leqslant x<R^{\alpha}$, then for $0 \leqslant \tau<x<R^{\alpha}$, we have:

$$
\begin{aligned}
D_{0}^{\alpha} Y(x) & =\frac{1}{\Gamma(r-\alpha)} \int_{0}^{x}(x-\tau)^{r-\alpha-1} Y^{(r)}(\tau) d \tau \\
& =\frac{1}{\Gamma(r-\alpha)} \int_{0}^{x}(x-\tau)^{r-\alpha-1}\left(\frac{d^{r}}{d \tau^{r}} \sum_{k=0}^{\infty} A_{k} \tau^{k}\right) d \tau \\
& =\sum_{k=0}^{\infty} A_{k}\left(\frac{1}{\Gamma(r-\alpha)} \int_{0}^{x}(x-\tau)^{r-\alpha-1}\left(\frac{d^{r}}{d \tau^{r}} \tau^{k}\right) d \tau\right)=\sum_{k=0}^{\infty} A_{k} D_{0}^{\alpha}\left(x^{k}\right) .
\end{aligned}
$$

Now by setting $x=t^{\alpha}, t \geqslant 0$ in Eq. (2.3), we get:

$$
\begin{aligned}
D_{0}^{\alpha} X(t)=D_{0}^{\alpha} Y\left(t^{\alpha}\right) & =\sum_{k=0}^{\infty} A_{k} D_{0}^{\alpha}\left(t^{k \alpha}\right), 0 \leqslant t^{\alpha}<R^{\alpha} \\
& =\sum_{k=1}^{\infty} A_{k} \frac{\Gamma(k \alpha+1)}{\Gamma((k-1) \alpha+1)} t^{(k-1) \alpha}, 0 \leqslant t<R .
\end{aligned}
$$

Likewise, we find that:

$$
\begin{aligned}
\mathrm{J}_{0}^{\alpha} Y(x) & =\frac{1}{\Gamma(\alpha)} \int_{0}^{x}(x-\tau)^{\alpha-1} Y(\tau) d \tau \\
& =\frac{1}{\Gamma(\alpha)} \int_{0}^{x}(x-\tau)^{\alpha-1}\left(\sum_{k=0}^{\infty} A_{k} \tau^{k}\right) d \tau \\
& =\sum_{k=0}^{\infty} A_{k}\left(\frac{1}{\Gamma(\alpha)} \int_{0}^{x}(x-\tau)^{\alpha-1}\left(\tau^{k}\right) d \tau\right)=\sum_{k=0}^{\infty} A_{k} J_{0}^{\alpha}\left(x^{k}\right) .
\end{aligned}
$$

Substitute $x=t^{\alpha}, t \geqslant 0$ in Eq. (2.4), then we get

$$
\begin{aligned}
J_{0}^{\alpha} X(t)=J_{0}^{\alpha} Y\left(t^{\alpha}\right) & =\sum_{k=0}^{\infty} A_{k} J_{0}^{\alpha}\left(t^{k \alpha}\right), 0 \leqslant t^{\alpha}<R^{\alpha} \\
& =\sum_{k=0}^{\infty} A_{k} \frac{\Gamma(k \alpha+1)}{\Gamma((k+1) \alpha+1)} t^{(k+1) \alpha}, 0 \leqslant t<R,
\end{aligned}
$$

and so the proof ends.

Theorem 2.12. Assume that $\mathrm{X}(\mathrm{t})=\left[\mathrm{x}_{\mathrm{ij}}(\mathrm{t})\right] \in \mathrm{M}_{\mathrm{m}, \mathrm{n}}$ has a MFPS representation at $\mathrm{t}_{0}$ of the form

$$
X(t)=\sum_{k=0}^{\infty} A_{k}\left(t-t_{0}\right)^{k \alpha}, 0 \leqslant r-1<\alpha \leqslant r, t_{0} \leqslant t<t_{0}+R .
$$

Then

(i) $\mathrm{X}(\mathrm{t})$ is analytic matrix function on $\left(\mathrm{t}_{0}, \mathrm{t}_{0}+\mathrm{R}\right)$;

(ii) if $\mathrm{X}(\mathrm{t})$ and $\mathrm{D}_{\mathrm{t}_{0}}^{\mathrm{k} \alpha} \mathrm{X}(\mathrm{t}) \in \mathrm{C}\left[\mathrm{t}_{0}, \mathrm{t}_{0}+\mathrm{R}\right), \mathrm{k}=0,1,2, \ldots$, then

$$
A_{k}=\frac{D_{t_{0}}^{k \alpha} X\left(t_{0}\right)}{\Gamma(k \alpha+1)}
$$

where $\mathrm{D}_{\mathrm{t}_{0}}^{\mathrm{k} \alpha}=\mathrm{D}_{\mathrm{t}_{0}}^{\alpha} \cdot \mathrm{D}_{\mathrm{t}_{0}}^{\alpha} \cdots \mathrm{D}_{\mathrm{t}_{0}}^{\alpha}(\mathrm{k}$-times $)$. That is

$$
X(t)=\sum_{k=0}^{\infty} \frac{D_{t_{0}}^{k \alpha} X\left(t_{0}\right)}{\Gamma(k \alpha+1)}\left(t-t_{0}\right)^{k \alpha}, 0 \leqslant r-1<\alpha \leqslant r, t_{0} \leqslant t<t_{0}+R,
$$

which is called the "Matrix fractional Taylor's series (MFTS)" about $\mathrm{t}_{0}$. 
As a special case, if $\alpha=1$, then we obtain the so-called "Matrix classical Taylor's series (MCTS)" about $\mathrm{t}_{0}$.

Proof.

(i). Since $Y(t)=\sum_{k=0}^{\infty} A_{k} t^{k}$ is analytic matrix function on $|t|<R^{\alpha}$ and $g(t)=\left(t-t_{0}\right)^{\alpha}$ is analytic function on $t_{0}<t<t_{0}+R, 0 \leqslant r-1<\alpha \leqslant r$, and so $(Y \circ g)(t)=X(t)$ is analytic matrix function on $\left(t_{0}, t_{0}+R\right)$.

(ii). Set $t=t_{0}$ in Eq. (2.5), then each term after the first one vanishes and thus we get: $A_{0}=X\left(t_{0}\right)$. Now by using part (5) Of Lemma 1.1, then for $t_{0} \leqslant t<t_{0}+R$, we have

$$
D_{t_{0}}^{\alpha} X(t)=\Gamma(\alpha+1) A_{1}+\frac{\Gamma(2 \alpha+1)}{\Gamma(\alpha+1)}\left(t-t_{0}\right)^{\alpha} A_{2}+\frac{\Gamma(3 \alpha+1)}{\Gamma(2 \alpha+1)}\left(t-t_{0}\right)^{2 \alpha} A_{3}+\cdots
$$

By substituting $t=t_{0}$ in Eq. (2.8), we obtain

$$
A_{1}=\frac{D_{t_{0}}^{\alpha} X\left(t_{0}\right)}{\Gamma(\alpha+1)} .
$$

Again, by applying Eq. (2.2) on Eq. (2.8), then for $t_{0} \leqslant t<t_{0}+R$, we have

$$
\mathrm{D}_{0}^{2 \alpha} X(\mathrm{t})=\Gamma(2 \alpha+1) \mathrm{A}_{2}+\frac{\Gamma(3 \alpha+1)}{\Gamma(\alpha+1)}\left(\mathrm{t}-\mathrm{t}_{0}\right)^{\alpha} \mathrm{A}_{3}+\frac{\Gamma(4 \alpha+1)}{\Gamma(2 \alpha+1)}\left(\mathrm{t}-\mathrm{t}_{0}\right)^{2 \alpha} \mathrm{A}_{4}+\cdots .
$$

Hence, if we put $t=t_{0}$ in Eq. (2.9), then we have

$$
A_{2}=\frac{D_{t_{0}}^{2 \alpha} X\left(t_{0}\right)}{\Gamma(2 \alpha+1)}
$$

By the same way, if we apply the operator $D_{t_{0}}^{\alpha}(\cdot) k$-times on the Eq. (2.5) and substitute $t=t_{0}$ in the resulting equation for each time, then it's easy to get the result as in Eq. (2.6) and by substituting the formula as in Eq. (2.6) into Eq. (2.5) we get the result as in Eq. (2.7).

Theorem 2.13. Assume that $\mathrm{X}(\mathrm{t})=\left[\mathrm{x}_{\mathrm{ij}}(\mathrm{t})\right] \in \mathrm{M}_{\mathrm{m}, \mathrm{n}}$ has a MFTS representation at $\mathrm{t}_{0}$ as in Eq. (2.5), $\mathrm{D}_{\mathrm{t}_{0}}^{\mathrm{k} \alpha} \mathrm{X}(\mathrm{t}) \in \mathrm{C}\left(\mathrm{t}_{0}, \mathrm{t}_{0}+\mathrm{R}\right), k=0,1,2, \ldots$ and $\mathrm{Y}(\mathrm{t})=\mathrm{X}\left(\left(\mathrm{t}-\mathrm{t}_{0}\right)^{1 / \alpha}+\mathrm{t}_{0}\right), \mathrm{t}_{0} \leqslant \mathrm{t}<\mathrm{t}_{0}+\mathrm{R}^{\alpha}$. Then

$$
D_{\mathrm{t}_{0}}^{k \alpha} X\left(\mathrm{t}_{0}\right)=\frac{\Gamma(k \alpha+1)}{k !} Y^{(\mathrm{k})}\left(\mathrm{t}_{0}\right) \text {. }
$$

Proof. Set $t=\left(x-t_{0}\right)^{1 / \alpha}+t_{0}, t_{0} \leqslant x<t_{0}+R^{\alpha}$ in Eq. (2.7), then we get

$$
Y(x)=X\left(\left(x-t_{0}\right)^{1 / \alpha}+t_{0}\right)=\sum_{k=0}^{\infty} \frac{D_{t_{0}}^{k \alpha} X\left(t_{0}\right)}{\Gamma(k \alpha+1)}\left(x-t_{0}\right)^{k}, t_{0} \leqslant x<t_{0}+R^{\alpha} .
$$

Also, the MCTS of $Y(x)$ about $t_{0}$ can be represented by

$$
Y(x)=\sum_{n=0}^{\infty} \frac{Y^{(n)}\left(t_{0}\right)}{n !}\left(x-t_{0}\right)^{n}, t_{0} \leqslant x<t_{0}+R^{\alpha} .
$$

Now by comparing the corresponding coefficients in Eq. (2.11) and (2.12), then we obtain the result as in Eq. (2.10).

Remark 2.14. The kth-partial sum of the MFTS of $X(t) \in M_{m, n}$ is

$$
\mathrm{T}_{k}(\mathrm{t})=\sum_{j=0}^{k} \frac{D_{\mathrm{t}_{0}}^{j \alpha} X\left(\mathrm{t}_{0}\right)}{\Gamma(j \alpha+1)}\left(t-\mathrm{t}_{0}\right)^{j \alpha},
$$

and $R_{k}(t)=X(t)-T_{k}(t)$ is called the "Remainder of the MFTS". 
Theorem 2.15. Let $X(t) \in M_{m, n}$ such that $X(t)$ and $D_{t_{0}}^{j \alpha} X(t) \in C\left(t_{0}, t_{0}+R\right), j=0,1,2, \ldots, k+1$ and $0<\alpha \leqslant 1$. Then the matrix function $\mathrm{X}(\mathrm{t})$ can be represented by

$$
X(t)=\sum_{j=0}^{k} \frac{\left(D_{t_{0}}^{j \alpha} X\right)\left(t_{0}\right)}{\Gamma(j \alpha+1)}\left(t-t_{0}\right)^{j \alpha}+J_{t_{0}}^{(k+1) \alpha} D_{t_{0}}^{(k+1) \alpha} X(t), \quad t_{0} \leqslant t \leqslant t_{0}+R .
$$

Proof. By applying Lemma 1.1, we get

$$
\begin{aligned}
J_{\mathfrak{t}_{0}}^{(k+1) \alpha} D_{\mathfrak{t}_{0}}^{(k+1) \alpha} X(t)= & J_{t_{0}}^{k \alpha}\left(\left(J_{t_{0}}^{\alpha} D_{t_{0}}^{\alpha}\right) D_{t_{0}}^{k \alpha} X(t)\right) \\
= & J_{t_{0}}^{k \alpha}\left(\left(J_{t_{0}} D_{t_{0}}\right) D_{t_{0}}^{k \alpha} X(t)\right) \\
= & J_{t_{0}}^{k \alpha}\left(D_{t_{0}}^{k \alpha} X(t)-D_{t_{0}}^{k \alpha} X\left(t_{0}\right)\right) \\
= & J_{t_{0}}^{k \alpha} D_{t_{0}}^{k \alpha} X(t)-J_{t_{0}}^{k \alpha}\left(D_{t_{0}}^{k \alpha} X\left(t_{0}\right)\right) \\
= & J_{t_{0}}^{(k-1) \alpha}\left(\left(J_{t_{0}} D_{t_{0}}\right) D_{t_{0}}^{(k-1) \alpha} X(t)\right)-\left(\frac{\left(D_{t_{0}}^{k \alpha} X\right)\left(t_{0}\right)}{\Gamma(k \alpha+1)}\left(t-t_{0}\right)^{k \alpha}\right) \\
= & J_{t_{0}}^{(k-1) \alpha}\left(D_{t_{0}}^{(k-1) \alpha} X(t)-D_{t_{0}}^{(k-1) \alpha} X\left(t_{0}\right)\right)-\left(\frac{\left(D_{t_{0}}^{k \alpha} X\right)\left(t_{0}\right)}{\Gamma(k \alpha+1)}\left(t-t_{0}\right)^{k \alpha}\right) \\
= & J_{t_{0}}^{(k-2) \alpha}\left(\left(J_{t_{0}} D_{t_{0}}\right) D_{t_{0}}^{(k-2) \alpha} X(t)\right)-\left(\frac{\left(D_{t_{0}}^{(k-1) \alpha} X\right)\left(t_{0}\right)}{\Gamma((k-1) \alpha+1)}\left(t-t_{0}\right)^{(k-1) \alpha}\right) \\
& -\left(\frac{\left(D_{t_{0}}^{k \alpha} X\right)\left(t_{0}\right)}{\Gamma(k \alpha+1)}\left(t-t_{0}\right)^{k \alpha}\right) .
\end{aligned}
$$

Now, we can get the result as in Eq. (2.13) after repeating previous procedure of calculations in Eq. (2.14) k-times.

Theorem 2.16. Let $X(t) \in M_{m, n}$ and for $0<\alpha \leqslant 1$,

$$
\left\|D_{t_{0}}^{(k+1) \alpha} X(t)\right\| \leqslant M, \quad t_{0} \leqslant t \leqslant \lambda .
$$

Then the reminder $R_{k}(t) \in M_{m, n}$ of the MFTS satisfies the inequality:

$$
\left\|R_{k}(t)\right\| \leqslant \frac{M}{\Gamma((k+1) \alpha+1)}\left(t-t_{0}\right)^{(k+1) \alpha}, \quad t_{0} \leqslant t \leqslant \lambda .
$$

Proof. Suppose that $D_{t_{0}}^{j \alpha} X(t)$ exist for $j=0,1,2, \ldots, k+1$ and since

$$
R_{k}(t)=X(t)-\sum_{j=0}^{k} \frac{D_{t_{0}}^{j \alpha} X\left(t_{0}\right)}{\Gamma(j \alpha+1)}\left(t-t_{0}\right)^{j \alpha},
$$

we get by applying Theorem 2.15

$$
R_{k}(t)=J_{t_{0}}^{(k+1) \alpha} D_{t_{0}}^{(k+1) \alpha} X(t) .
$$

Thus for $t_{0} \leqslant \tau \leqslant t \leqslant \lambda$, we have

$$
\begin{aligned}
\left\|R_{k}(t)\right\| & =\left\|J_{t_{0}}^{(k+1) \alpha} D_{t_{0}}^{(k+1) \alpha} X(t)\right\| \\
& =\left\|\frac{1}{\Gamma((k+1) \alpha)} \int_{t_{0}}^{t}(t-\tau)^{(k+1) \alpha-1} D_{\mathfrak{t}_{0}}^{(k+1) \alpha} X(\tau) d \tau\right\|
\end{aligned}
$$




$$
\begin{aligned}
& \leqslant \frac{1}{\Gamma((k+1) \alpha)} \int_{\mathrm{t}_{0}}^{\mathrm{t}}\left|(\mathrm{t}-\tau)^{(\mathrm{k}+1) \alpha-1}\right||| \mathrm{D}_{\mathrm{t}_{0}}^{(\mathrm{k}+1) \alpha} X(\tau) \| \mathrm{d} \tau \\
& \leqslant \frac{1}{\Gamma((\mathrm{k}+1) \alpha)} \int_{\mathrm{t}_{0}}^{\mathrm{t}}\left|(\mathrm{t}-\tau)^{(\mathrm{k}+1) \alpha-1}\right| \mathrm{Md} \tau \\
& =\frac{M}{\Gamma((\mathrm{k}+1) \alpha)} \int_{\mathrm{t}_{0}}^{\mathrm{t}}(\mathrm{t}-\tau)^{(\mathrm{k}+1) \alpha-1} \mathrm{~d} \tau \\
& =\frac{M}{\Gamma((\mathrm{k}+1) \alpha+1)}\left(\mathrm{t}-\mathrm{t}_{0}\right)^{(\mathrm{k}+1) \alpha} .
\end{aligned}
$$

\section{Frobenius norm approximation of matrix fractional derivatives and integrals}

In this section, we present the Frobenius norm approximation for the matrix fractional derivatives and integrals of the matrix function $X(t) \in M_{m, n}$ at a given point based on our results as obtained in Section 2 (Theorems 2.11, 2.12, 2.13 and MFTS method). Here we use Mathematica software packages for getting the numerical computations.

Prior anything, we recall the definition of the Frobenius norm contained in the following formula

$$
\|X(t)\|_{F}=\sqrt{\left(\sum_{i=1}^{m} \sum_{j=1}^{n}\left|x_{i j}(t)\right|^{2}\right)}, X(t)=\left[x_{i j}(t)\right] \in M_{m, n} .
$$

Problem 3.1. Given the following matrix function:

$$
X(t)=\frac{I_{4}}{1-t^{\alpha}} \in M_{4 \times 4}, \quad \alpha>0, t \geqslant 0,
$$

where $\mathrm{I}_{4}$ is an identity matrix of order 4 .

The MFTS of $X(t)$ about $t=0$ is written by

$$
X(t)=\sum_{k=0}^{\infty} \frac{D_{0}^{k \alpha} X(0)}{\Gamma(k \alpha+1)} t^{k \alpha}, \alpha>0, t \geqslant 0
$$

which is called the "Matrix fractional Maclaurin's series (MFMS)" of X(t). According to Theorem 2.13, we have

$$
D_{0}^{k \alpha} X(0)=\frac{\Gamma(k \alpha+1)}{k !} Y^{(k)}(0), \alpha>0, t \geqslant 0,
$$

where

$$
\mathrm{Y}(\mathrm{t})=X\left(\mathrm{t}^{1 / \alpha}\right)=\frac{\mathrm{I}_{4}}{1-\mathrm{t}^{\prime}}, \mathrm{Y}^{(\mathrm{k})}(0)=\mathrm{I}_{4} \mathrm{k} !
$$

That is, the MFMS of $X(t)$ can be represented as follows

$$
X(t)=\sum_{k=0}^{\infty} I_{4} t^{k \alpha}, \alpha>0, t \geqslant 0,
$$

which is a geometric MFPS with ratio $t^{\alpha}$ and converges for each $0 \leqslant t^{\alpha}<1$, and so for each $0 \leqslant t<1$.

Now according to Theorem 2.11, we can approximate the matrix fractional derivative $\left\|D_{0}^{\alpha} X(t)\right\|_{F}$ and the matrix fractional integral $\left\|J_{0}^{\alpha} X(t)\right\|_{F}$ of a matrix function $X(t)$ on $0 \leqslant t<1$, beginningwith $\left\|D_{0}^{\alpha} X(t)\right\|_{F}$ 
which can be bounded by the $\mathrm{m}^{\text {th }}$-partial sum of its expansion by

$$
\begin{aligned}
\left\|D_{0}^{\alpha} X(t)\right\|_{F} & \cong\left\|I_{4} \sum_{k=1}^{m} \frac{\Gamma(k \alpha+1)}{\Gamma((k-1) \alpha+1)} t^{(k-1) \alpha}\right\|_{F}, 0 \leqslant t<1, \\
& \leqslant\left\|I_{4}\right\|_{F} \sum_{k=1}^{m}\left|\frac{\Gamma(k \alpha+1)}{\Gamma((k-1) \alpha+1)} t^{(k-1) \alpha}\right|, \\
& =2 \sum_{k=1}^{m} \frac{\Gamma(k \alpha+1)}{\Gamma((k-1) \alpha+1)} t^{(k-1) \alpha} .
\end{aligned}
$$

Table 1 shows the numerical values of $\left\|D_{0}^{\alpha} X(t)\right\|_{F}$ for distinct values of $t$ and $\alpha$ on $0 \leqslant t<1$ in step of 0.1 when $m=10$.

\begin{tabular}{ccccc} 
Table 1: & Numerical values of $\left\|D_{0}^{\alpha} X(t)\right\|_{F}$ when $\mathrm{m}=10$. \\
\hline $\mathrm{t}$ & $\alpha=1 / 2$ & $\alpha=3 / 4$ & $\alpha=3 / 2$ & $\alpha=2$ \\
\hline 0.0 & 1.77245 & 1.83813 & 2.65868 & 4.00000 \\
0.1 & 2.89754 & 2.50723 & 2.9625 & 4.24611 \\
0.2 & 3.83615 & 3.23901 & 3.62818 & 5.06366 \\
0.3 & 4.99905 & 4.22712 & 4.77134 & 6.74124 \\
0.4 & 6.52266 & 5.65090 & 6.74233 & 9.98811 \\
0.5 & 8.55521 & 7.79886 & 10.3588 & 16.5913 \\
0.6 & 11.2710 & 11.1383 & 17.6872 & 31.6794 \\
0.7 & 14.8752 & 16.4033 & 34.4080 & 72.7418 \\
0.8 & 19.6062 & 24.7069 & 76.7787 & 208.884 \\
0.9 & 25.7382 & 37.6799 & 190.973 & 731.952 \\
\hline
\end{tabular}

Similarly, the $\mathrm{m}^{\text {th }}$-partial sum expansion for approximating $\left\|J_{0}^{\alpha} X(t)\right\|_{F}$ can be obtained by Theorem 2.11 as follows

$$
\begin{aligned}
\left\|J_{0}^{\alpha} X(t)\right\|_{F} & \cong\left\|I_{4} \sum_{k=0}^{m} \frac{\Gamma(k \alpha+1)}{\Gamma((k+1) \alpha+1)} \mathrm{t}^{(k+1) \alpha}\right\|_{F}, \alpha>0,0 \leqslant t<1, \\
& \leqslant \sum_{k=0}^{m} \frac{2 \Gamma(k \alpha+1)}{\Gamma((k+1) \alpha+1)} \mathrm{t}^{(k+1) \alpha} .
\end{aligned}
$$

Table 2 shows the numerical values of $\left\|J_{0}^{\alpha} X(t)\right\|_{F}$ for distinct values of $t$ and $\alpha$ on $0 \leqslant t<1$ when $m=10$.

\begin{tabular}{cccll} 
Table 2: & Numerical values of $\left\|J_{0}^{\alpha} \mathrm{X}(\mathrm{t})\right\|_{\mathrm{F}}$ when $\mathrm{m}=10$. \\
\hline $\mathrm{t}$ & $\alpha=1 / 2$ & $\alpha=3 / 4$ & $\alpha=3 / 2$ & $\alpha=2$ \\
\hline 0.0 & 0.00000 & 0.00000 & 0.0000 & 0.00000 \\
0.1 & 0.24349 & 0.05059 & 0.00045 & 0.00002 \\
0.2 & 0.57892 & 0.16072 & 0.00372 & 0.00027 \\
0.3 & 1.01824 & 0.33195 & 0.01310 & 0.00140 \\
0.4 & 1.59074 & 0.57880 & 0.03280 & 0.00457 \\
0.5 & 2.33971 & 0.92714 & 0.06857 & 0.01162 \\
0.6 & 3.32452 & 1.41993 & 0.12897 & 0.02549 \\
0.7 & 4.62349 & 2.12725 & 0.22806 & 0.05087 \\
0.8 & 6.33708 & 3.16197 & 0.39193 & 0.09609 \\
0.9 & 8.59139 & 4.70257 & 0.67637 & 0.17828 \\
\hline
\end{tabular}


Problem 3.2. Given the following MM-LF of $A \in M_{n}$ :

$$
E_{\alpha}\left(A t^{\alpha}\right)=\sum_{k=0}^{\infty} \frac{A^{k} t^{k \alpha}}{\Gamma(k \alpha+1)}, \quad \alpha>0, t \geqslant 0 .
$$

Since the general solutions of many fractional differential system are represented in terms of $E_{\alpha}\left(A t^{\alpha}\right)$, then we need here to approximate: $\left\|D_{0}^{\alpha}\left(E_{\alpha}\left(A t^{\alpha}\right)\right)\right\|_{F}$ and $\left\|J_{0}^{\alpha}\left(E_{\alpha}\left(A t^{\alpha}\right)\right)\right\|_{F}$, respectively, for $m^{\text {th }}$-partial sums based on Theorem 2.11 as follow

$$
\begin{gathered}
\left\|D_{0}^{\alpha}\left(E_{\alpha}\left(A t^{\alpha}\right)\right)\right\|_{F}=\left\|A E_{\alpha}\left(A t^{\alpha}\right)\right\|_{F} \cong\left\|\sum_{k=0}^{m} \frac{A^{k+1} t^{k \alpha}}{\Gamma(k \alpha+1)}\right\|_{F} \leqslant \sum_{k=0}^{m} \frac{\|A\|_{F}^{k+1} t^{k \alpha}}{\Gamma(k \alpha+1)}, \alpha>0, t \geqslant 0, \\
\left\|J_{0}^{\alpha}\left(E_{\alpha}\left(A t^{\alpha}\right)\right)\right\|_{F} \cong\left\|\sum_{k=0}^{m} \frac{A^{k} t^{(k+1) \alpha}}{\Gamma((k+1) \alpha+1)}\right\|_{F} \leqslant \sum_{k=0}^{m} \frac{\|A\|_{F}^{k} t^{(k+1) \alpha}}{\Gamma((k+1) \alpha+1)}, \alpha>0, t \geqslant 0 .
\end{gathered}
$$

To show the validity of our MFPS representation for the approximating $E_{\alpha}\left(A t^{\alpha}\right)$, consider

$$
A=\left[\begin{array}{cc}
\frac{1}{4} & \frac{1}{4} \\
\frac{1}{4} & -\frac{1}{4}
\end{array}\right] .
$$

\begin{tabular}{|c|c|c|c|c|}
\hline $\mathrm{t}$ & $\alpha=1 / 2$ & $\alpha=3 / 4$ & $\alpha=3 / 2$ & $\alpha=2$ \\
\hline 0.0 & 0.50000 & 0.50000 & 0.50000 & 0.50000 \\
\hline 0.4 & 0.74338 & 0.66409 & 0.54893 & 0.52013 \\
\hline 0.8 & 0.89951 & 0.81536 & 0.64568 & 0.58216 \\
\hline 1.2 & 1.05385 & 0.98370 & 0.78606 & 0.69106 \\
\hline 1.6 & 1.21501 & 1.17624 & 0.97661 & 0.85562 \\
\hline 2.0 & 1.38709 & 1.39880 & 1.22860 & 1.08909 \\
\hline 2.4 & 1.57296 & 1.65743 & 1.55775 & 1.41027 \\
\hline 2.8 & 1.77504 & 1.95887 & 1.98495 & 1.84502 \\
\hline 3.2 & 1.99563 & 2.31081 & 2.53745 & 2.42837 \\
\hline 3.6 & 2.23704 & 2.72215 & 3.25056 & 3.20728 \\
\hline 4.0 & 2.50164 & 3.20322 & 4.16986 & 4.24448 \\
\hline
\end{tabular}

Hence, the numerical values of $\left\|D_{0}^{\alpha}\left(E_{\alpha}\left(A t^{\alpha}\right)\right)\right\|_{F}$ and $\left\|J_{0}^{\alpha}\left(E_{\alpha}\left(A t^{\alpha}\right)\right)\right\|_{F}$ for distinct values of $\alpha$ and $t$ on $0 \leqslant t \leqslant 4$ when $m=10$ are given, respectively, as in Tables 3 and 4 .

Table 4: Numerical Values of $\left\|J_{0}^{\alpha}\left(E_{\alpha}\left(A t^{\alpha}\right)\right)\right\|_{F}$ when $m=10$.

\begin{tabular}{lllll}
\hline $\mathrm{t}$ & $\alpha=1 / 2$ & $\alpha=3 / 4$ & $\alpha=3 / 2$ & $\alpha=2$ \\
\hline 0.0 & 0.00000 & 0.00000 & 0.00000 & 0.00000 \\
0.4 & 0.97353 & 0.65637 & 0.19572 & 0.08054 \\
0.8 & 1.59803 & 1.26143 & 0.58273 & 0.32863 \\
1.2 & 2.21540 & 1.93481 & 1.14425 & 0.76425 \\
1.6 & 2.86007 & 2.70494 & 1.90645 & 1.42249 \\
2.0 & 3.54851 & 3.59519 & 2.91440 & 2.35637 \\
2.4 & 4.29224 & 4.62975 & 4.23100 & 3.64108 \\
2.8 & 5.10113 & 5.83555 & 5.93980 & 5.38009 \\
3.2 & 5.98457 & 7.24346 & 8.14981 & 7.71346 \\
3.6 & 6.95207 & 8.88915 & 11.0023 & 10.8291 \\
4.0 & 8.01350 & 10.8142 & 14.6794 & 14.9779 \\
\hline
\end{tabular}




\section{MFPS solutions of some linear and non-linear MFDEs}

This section focuses on the general exact (numerical) solutions of four important and interesting linear and non-linear MFDEs by using the efficient MFPS technique. Also, a new technique is applied on the non-linear MFDEs as in the last two problems.

Problem 4.1. Given the following linear MFDEs:

$$
\begin{aligned}
\mathrm{D}_{0}^{2 \alpha} X(\mathrm{t}) & =-\mathrm{W}^{2} \mathrm{X}(\mathrm{t}), 0<\alpha \leqslant 1, \mathrm{t} \geqslant 0, \\
X(0) & =\mathrm{P}_{0}, \mathrm{D}_{0}^{\alpha} \mathrm{X}(0)=\mathrm{P}_{1},
\end{aligned}
$$

where $W \in M_{m}, P_{0}$ and $P_{1} \in M_{m, n}$ are real or complex constant matrices and $X(t) \in M_{m, n}$.

According to MFMS method, assume that the MFPS solution $X(t)$ of Eq. (4.1) as follows:

$$
X(t)=\sum_{k=0}^{\infty} A_{k} t^{k \alpha}
$$

Now, by applying the operator $\mathrm{D}_{0}^{\alpha}$ two-times on Eq. (4.3), we have

$$
\begin{aligned}
D_{0}^{\alpha} X(t) & =\sum_{k=1}^{\infty} A_{k} \frac{\Gamma(k \alpha+1)}{\Gamma((k-1) \alpha+1)} t^{(k-1) \alpha} \\
D_{0}^{2 \alpha} X(t) & =\sum_{k=2}^{\infty} A_{k} \frac{\Gamma(k+1)}{\Gamma((k-2) \alpha+1)} t^{(k-2) \alpha}=\sum_{k=0}^{\infty} A_{k+2} \frac{\Gamma((k+2) \alpha+1)}{\Gamma(k \alpha+1)} t^{k \alpha} .
\end{aligned}
$$

Substitute Eqs. (4.3) and (4.4) into Eq. (4.1), yields that:

$$
\sum_{k=0}^{\infty} A_{k+2} \frac{\Gamma((k+2) \alpha+1)}{\Gamma(k \alpha+1)} t^{k \alpha}+W^{2} \sum_{k=0}^{\infty} A_{k} t^{k \alpha}=0 .
$$

This formula leads to the following recurrence relation:

$$
A_{k+2}=\frac{-\Gamma(k \alpha+1)}{\Gamma((k+2) \alpha+1)} W^{2} A_{k}, k=0,1,2, \ldots
$$

By using the initial conditions as in Eq. (4.2), we have

$$
A_{0}=P_{0}, A_{1}=\frac{1}{\Gamma(\alpha+1)} P_{1} .
$$

Now, the other coefficients of $t^{k \alpha}$ can be partitioned as follows

1. For the terms of even indices are:

$$
A_{2}=\frac{-1}{\Gamma(2 \alpha+1)} W^{2} P_{0}, A_{4}=\frac{1}{\Gamma(4 \alpha+1)} W^{4} P_{0}, \ldots
$$

2. For the terms of odd indices are:

$$
A_{3}=\frac{-1}{\Gamma(3 \alpha+1)} W^{2} P_{1}, A_{5}=\frac{1}{\Gamma(5 \alpha+1)} W^{4} P_{1}, \ldots
$$


Thus, we can obtain the series solution as follows

$$
X(t)=\sum_{k=0}^{\infty} W^{2 k} P_{0} \frac{(-1)^{k}}{\Gamma(2 k \alpha+1)} t^{2 k \alpha}+\sum_{k=0}^{\infty} W^{2 k} P_{1} \frac{(-1)^{k}}{\Gamma((2 k+1) \alpha+1)} t^{(2 k+1) \alpha} .
$$

This solution can be represented in the term of the MM-LF as an exact solution by

$$
X(t)=E_{2 \alpha}\left(-W^{2} t^{2 \alpha}\right) P_{0}+t^{\alpha} E_{2 \alpha,(\alpha+1)}\left(-W^{2} t^{2 \alpha}\right) P_{1} .
$$

Problem 4.2. Given the following composite linear MFDEs:

$$
\begin{aligned}
D_{0}^{2} X(t)+D_{0}^{1 / 2} X(t)+X(t) & =8 B, t \geqslant 0, \\
X(0)=X^{\prime}(0) & =0
\end{aligned}
$$

where $B$ and $X(t) \in M_{m, n}$.

According to the MFMS method, suppose that the MFPS solution $X(t)$ of Eq. (4.5) as follows:

$$
X(t)=\sum_{k=0}^{\infty} A_{k} t^{\frac{k}{2}}
$$

The MFMS requires to find the fractional derivatives: $D_{0}^{1 / 2} X(t), D_{0}^{1} X(t)$, and $D_{0}^{2} X(t)$. However, it is easy to find them by using Eq. (2.2) as follow

$$
\begin{aligned}
D_{0}^{1 / 2} X(t) & =\sum_{k=1}^{\infty} A_{k} \frac{\Gamma\left(\frac{k}{2}+1\right)}{\Gamma\left(\frac{k-1}{2}+1\right)} t^{\frac{k-1}{2}}=\sum_{k=0}^{\infty} A_{k+1} \frac{\Gamma\left(\frac{k+1}{2}+1\right)}{\Gamma\left(\frac{k}{2}+1\right)} t^{\frac{k}{2}} \\
D_{0}^{1} X(t) & =\frac{d X}{d t}=A_{1} t^{\frac{-1}{2}}+A_{2}+\sum_{k=3}^{\infty} A_{n} \frac{k}{2} t^{\frac{k-2}{2}} \\
D_{0}^{2} X(t) & =\frac{d^{2} X}{d t^{2}}=\frac{-1}{2} A_{1} t^{\frac{-3}{2}}+\frac{3}{4} A_{3} t^{\frac{-3}{2}}+\sum_{k=4}^{\infty} k_{n} \frac{k}{2}\left(\frac{k}{2}-1\right) t^{\frac{k-4}{2}}
\end{aligned}
$$

Since $t \geqslant 0$, then $A_{1}$ and $A_{3}$ must be zeros and by using the initial conditions as in Eq. (4.6), we get $A_{0}=A_{2}=0$.

Now, the new representation form of the solution is obtained by:

$$
\begin{aligned}
X(t) & =\sum_{k=4}^{\infty} A_{k} t^{\frac{k}{2}} \\
D_{0}^{1 / 2} X(t) & =A_{4} \frac{2}{\Gamma\left(\frac{5}{2}\right)} t^{\frac{3}{2}}+\sum_{k=4}^{\infty} A_{k+1} \frac{\Gamma\left(\frac{k+1}{2}+1\right)}{\Gamma\left(\frac{k}{2}+1\right)} t^{\frac{k}{2}} \\
D_{0}^{2} y(t) & =2 A_{4}+\frac{15}{4} A_{5} t^{\frac{1}{2}}+6 A_{6} t+\frac{35}{4} A_{7} t^{\frac{3}{2}}+\sum_{k=4}^{\infty} A_{k+4} \frac{k+4}{2}\left(\frac{k+4}{2}-1\right) t^{\frac{k}{2}} .
\end{aligned}
$$

Substitute the expansion formulas above into Eq. (4.5), we obtain

$$
\begin{aligned}
A_{4} & =4 B, A_{5}=A_{6}=0, A_{7}=-\frac{128 B}{(105 \sqrt{\pi})} \\
A_{k+4} & =\frac{-4}{(k+2)(k+4)}\left(A_{k}+A_{k+1} \times \frac{\left(\frac{k+1}{2}+1\right)}{\Gamma\left(\frac{k}{2}+1\right)}\right), \quad k \geqslant 4 .
\end{aligned}
$$


So that the 15th-truncated series approximation of $X(t)$ is represented by

$$
X_{15}(t)=4 B t^{2}-\frac{128 B}{105 \sqrt{\pi}} t^{\frac{7}{2}}-\frac{B}{3} t^{4}+\frac{B}{15} t^{5}+\frac{1024 B}{10395 \sqrt{\pi}} t^{\frac{11}{2}}+\frac{B}{90} t^{6}-\frac{1024 B}{135135 \sqrt{\pi}} t^{\frac{13}{2}}-\frac{B}{210} t^{7}-\frac{2048 B}{675675 \sqrt{\pi}} t^{\frac{15}{2}} .
$$

In order to examine the approximation solution in Eq. (4.7), we need to calculate the residual error function $(\operatorname{Res}(t))$ with respect to the Frobenius norm for different values $t$ on $0 \leqslant t \leqslant 1$ in step of 0.2 , where the residual error function is defined as follows

$$
\operatorname{Res}(t)=\left\|D_{0}^{2} X(t)+D_{0}^{\frac{1}{2}} X(t)+X(t)-8 B\right\|_{F},
$$

and here, we will take a fixed numerical values for the matrix $B$ as follows:

$$
\mathrm{B}=\left[\begin{array}{ccc}
\frac{1}{4} & 0 & -\frac{1}{4} \\
0 & \frac{1}{4} & -\frac{1}{4}
\end{array}\right]
$$

Table 5 shows that the 15th-numerical values of $\|X(t)\|_{F},\left\|D_{0}^{1 / 2} X(t)\right\|_{F}$, and $\left\|D_{0}^{2} X(t)\right\|_{F}$ with Res $(t)$. It indicates that the numerical solution of the problem as in Eq. (4.7) is more accurate at the beginning of the values of the interval. In fact, we can say that the MFPS method is efficient to obtain a good accuracy of the solution.

\begin{tabular}{cclll} 
Table 5: 15th-Numerical values of $\|\mathrm{X}(\mathrm{t})\|_{\mathrm{F}},\left\|\mathrm{D}_{0}^{1 / 2} \mathrm{X}(\mathrm{t})\right\|_{\mathrm{F}},\left\|\mathrm{D}_{0}^{2} \mathrm{X}(\mathrm{t})\right\|_{\mathrm{F}}$ and Res $(\mathrm{t})$. \\
\hline $\mathrm{t}$ & $\|\mathrm{X}(\mathrm{t})\|_{\mathrm{F}}$ & $\left\|\mathrm{D}_{0}^{1 / 2} \mathrm{X}(\mathrm{t})\right\|_{\mathrm{F}}$ & $\left\|\mathrm{D}_{0}^{2} \mathrm{X}(\mathrm{t})\right\|_{\mathrm{F}}$ & $\operatorname{Res}(\mathrm{t})$ \\
\hline 0.0 & 0.00000 & 0.00000 & 0.00000 & 0.00000 \\
0.2 & 0.07852 & 0.26265 & 3.65883 & $3.10574 \times 10^{-7}$ \\
0.4 & 0.30235 & 0.70661 & 2.99101 & $3.08381 \times 10^{-5}$ \\
0.6 & 0.64523 & 1.21006 & 2.14425 & $4.60852 \times 10^{-4}$ \\
0.8 & 1.07361 & 1.70471 & 1.21851 & $3.16383 \times 10^{-3}$ \\
1.0 & 1.55075 & 2.14109 & 0.29399 & $1.41674 \times 10^{-2}$ \\
\hline
\end{tabular}

Problem 4.3. Given the following nonlinear MFDE:

$$
\begin{aligned}
& D_{0}^{\alpha} X(t)=X^{2}(t)+I_{n}, \quad r-1<\alpha \leqslant r, t \geqslant 0, \\
& X^{(i)}(0)=0, \quad i=0,1, \ldots, r-1,
\end{aligned}
$$

where $I_{n}$ is an identity matrix and $X(t) \in M_{n}$.

Similarly to the previous problems, let the MFPS solution of Eqs. (4.8) and (4.9) as:

$$
X(t)=\sum_{k=0}^{\infty} A_{k} t^{k \alpha} .
$$

Based on Eq. (4.9), we get $A_{0}=0$ and so Eq. (4.10) becomes:

$$
X(t)=\sum_{k=1}^{\infty} A_{k} t^{k \alpha} .
$$

In general, it is not easy to find the coefficients $A_{k}$ from the recurrence relation corresponding to the MFPS representation for nonlinear MFDEs. Therefore, we use a new technique in this problem for finding $A_{k}$ by defining the so-called $\alpha$ mth-order MFDE as follows:

$$
D_{0}^{\alpha m}\left(D_{0}^{\alpha} X(t)-X^{2}(t)-I_{n}\right)=0, m=0,1,2, \ldots
$$


Note that when $m=0$, then Eq. (4.12) is reduced to Eq. (4.8). So, the MFMS representation in Eq. (4.11) is a solution for the $\alpha \mathrm{mth}$-order MFDE as in Eq. (4.12). That is:

$$
D_{0}^{\alpha(m+1)}\left(\sum_{k=1}^{\infty} A_{k} t^{k \alpha}\right)-D_{0}^{\alpha m}\left(\sum_{k=1}^{\infty} A_{k} t^{k \alpha}\right)^{2}-D_{0}^{\alpha m}\left(I_{n}\right)=0, m=0,1,2, \ldots
$$

Based on Eq. (2.2), then Eq. (4.13) becomes

$$
\sum_{k=m+1}^{\infty} A_{k} \frac{\Gamma(k \alpha+1)}{\Gamma((k-m-1) \alpha+1)} t^{(k-m-1) \alpha}-\sum_{k=m}^{\infty}\left(\sum_{j=0}^{k} A_{j} A_{k-j}\right) \frac{\Gamma(k \alpha+1)}{\Gamma((k-m) \alpha+1)} t^{(k-m) \alpha}=\chi_{m},
$$

where $x_{m}=I_{n}$ if $m=0$ and $\chi_{m}=0$ if $m \geqslant 1$.

Hence by using Theorems (2.7) and (2.11), the $\alpha \mathrm{mth}$ derivative of the MFMS representation, Eq. (4.11) converges at least at $t=0$, for $m=0,1,2, \ldots$

Now, by setting $t=0$ in Eq. (4.14), we get the following values of the coefficients $A_{k}$ of $t^{k \alpha}$ :

$$
A_{0}=0, A_{1}=\frac{I}{\Gamma(\alpha+1)}, A_{m+1}=\frac{\Gamma(m \alpha+1)}{\Gamma((1+m) \alpha+1)} \sum_{j=0}^{m} A_{j} A_{m-j} \text { for } m=1,2, \ldots
$$

Thus, the general expansion solution of Eqs. (4.8) and (4.9) is obtained as follows:

$$
\mathrm{X}(\mathrm{t})=\frac{\mathrm{I}_{\mathrm{n}}}{\Gamma(\alpha+1)} \mathrm{t}^{\alpha}+\frac{\Gamma(2 \alpha+1) \mathrm{I}_{\mathrm{n}}}{(\Gamma(\alpha+1))^{2} \Gamma(3 \alpha+1)} \mathrm{t}^{3 \alpha}+2 \frac{\Gamma(2 \alpha+1) \Gamma(4 \alpha+1) \mathrm{I}_{\mathrm{n}}}{(\Gamma(\alpha+1))^{3} \Gamma(3 \alpha+1) \Gamma(5 \alpha+1)} \mathrm{t}^{5 \alpha}+\ldots
$$

In particular, if $\alpha=1$, then the Eqs. (4.8) and (4.9) become:

$$
X^{\prime}(t)=X^{2}(t)+I_{n}, X(0)=0, t \geqslant 0,
$$

and when applying Eq. (4.15), then the CPS solution of Eq. (4.16) is presented by:

$$
X(t)=\left(t+\frac{t^{3}}{3}+\frac{2 t^{5}}{15}+\frac{17 t^{7}}{315}+\frac{62 t^{9}}{2835}+\frac{1382 t^{11}}{155925}+\cdots\right) I_{n}=I_{n} \tan t .
$$

In order to examine the approximate solution as in Eq. (4.15), Table 6 shows that the 15th-approximate of $\|X(t)\|_{F}$ with Res $(t)$ for different values of $\alpha$ and $t$ on $0 \leqslant t \leqslant 1$ by considering the identity matrix of order 9 and defining the residual error function of Problem 4.3 with respect to Frobenius norm by:

$$
\operatorname{Res}(t)=\left\|D_{0}^{\alpha} X(t)-X^{2}(t)-I_{9}\right\|_{F} .
$$

Table 6: 15th-numerical values of $\|X(t)\|_{F}$ with $\operatorname{Res}(t)$.

\begin{tabular}{lllll}
\hline $\mathbf{t}$ & $\|\mathbf{X}(\mathbf{t} ; \boldsymbol{\alpha}=\mathbf{1 . 5})\|_{\mathbf{F}}$ & $\boldsymbol{R e s}(\mathbf{t} ; \boldsymbol{\alpha}=\mathbf{1 . 5})$ & $\|\mathbf{X}(\mathbf{t} ; \boldsymbol{\alpha}=\mathbf{2 . 5})\|_{\mathbf{F}}$ & $\operatorname{Res}(\mathbf{t} ; \boldsymbol{\alpha = 2 . 5})$ \\
\hline 0.0 & 0.00000 & 0.00000 & 0.000000 & 0.00000 \\
0.2 & 0.20199 & $2.034437 \times 10^{-17}$ & 0.016149 & $3.109055 \times 10^{-16}$ \\
0.4 & 0.57409 & $4.370361 \times 10^{-17}$ & 0.091350 & $1.252591 \times 10^{-15}$ \\
0.6 & 1.06871 & $2.850815 \times 10^{-13}$ & 0.251775 & $7.275543 \times 10^{-16}$ \\
0.8 & 1.68902 & $2.897717 \times 10^{-10}$ & 0.517173 & $1.022700 \times 10^{-15}$ \\
1.0 & 2.46753 & $6.341391 \times 10^{-8}$ & 0.905028 & $1.998026 \times 10^{-16}$ \\
\hline
\end{tabular}

Table 6 provides that the numerical values for the convergence of the MFPS technique and the results shown here confirm that our new method is very efficient by using only a few approximation terms and higher accuracy can be achieved by computing more components of the solution. 
Problem 4.4. Given the following composite nonlinear MFDE:

$$
\begin{aligned}
D_{0}^{2 \alpha} X(t) & =\left(D_{0}^{\alpha} X(t)\right)^{2}+I_{n}, \frac{1}{2}<\alpha \leqslant 1, t \geqslant 0, \\
X(0) & =C_{0}, \quad D_{0}^{\alpha} X(0)=C_{1},
\end{aligned}
$$

where $I_{n}$ is an identity matrix of order $n$, and $C_{0}, C_{1}, X(t) \in M_{n}$.

Again, the MFPS solution of Eqs. (4.17) and (4.18) can be assumed as follows:

$$
X(t)=\sum_{k=0}^{\infty} A_{k} t^{k \alpha}
$$

Thus, the $\alpha$ mth-order MFDE of Eq. (4.17) is:

$$
D_{0}^{m \alpha}\left(\left(D_{0}^{2 \alpha} \sum_{k=0}^{\infty} A_{k} t^{k \alpha}\right)-\left(D_{0}^{\alpha} \sum_{k=0}^{\infty} A_{k} t^{k \alpha}\right)^{2}-I_{n}\right)=0, m=0,1,2, \ldots
$$

Based on Eq. (4.17) and using Cauchy product for infinite series, then Eq. (4.19) becomes:

$$
\mathrm{D}_{0}^{\mathrm{m} \alpha}\left(\sum_{\mathrm{k}=2}^{\infty} \mathrm{A}_{\mathrm{k}} \frac{\Gamma(\mathrm{k} \alpha+1)}{\Gamma((\mathrm{k}-2) \alpha+1)} \mathrm{t}^{(\mathrm{k}-2) \alpha}-\sum_{\mathrm{k}=0}^{\infty} \mathrm{B}_{\mathrm{k}} \mathrm{t}^{\mathrm{k} \alpha}-\mathrm{I}_{\mathrm{n}}\right)=0,
$$

where

$$
B_{k}=\sum_{j=0}^{k} A_{j+1} A_{k-j+1} \frac{\Gamma((j+1) \alpha+1)}{\Gamma(j \alpha+1)} \frac{\Gamma((k-j+1) \alpha+1)}{\Gamma((k-j) \alpha+1)} .
$$

In fact, Eq. (4.20) can be easily reduced into the following equivalent form:

$$
\sum_{k=m+2}^{\infty} A_{k} \frac{\Gamma(k \alpha+1)}{\Gamma((k-m-2) \alpha+1)} \mathrm{t}^{(k-m-2) \alpha}-\sum_{k=m}^{\infty} B_{k} \frac{\Gamma(k \alpha+1)}{\Gamma((k-m) \alpha+1)} t^{(k-m) \alpha}=\chi_{m},
$$

where $x_{m}=I_{n}$ if $m=0$ and $x_{m}=0$ if $m \geqslant 1$.

However, setting $t=0$ in Eq. (4.21) gives the values of $A_{k}$, the coefficient of $t^{k \alpha}$, follows as

$$
A_{0} \text { and } A_{1} \text { are arbitrary, } A_{2}=\frac{I+C_{1}^{2}(\Gamma(\alpha+1))^{2}}{\Gamma(2 \alpha+1)}, \quad A_{m+2}=\frac{\Gamma(m \alpha+1)}{\Gamma((2+m) \alpha+1)} B_{m}, m=1,2, \ldots
$$

Now, by simple computations, we get the exact solution of Eqs. (4.17) and (4.18) which are expanded in the following power series solution

$$
X(t)=C_{0}+C_{1} t^{\alpha}+\frac{I_{n}+C_{1}^{2}(\Gamma(\alpha+1))^{2}}{\Gamma(2 \alpha+1)} t^{2 \alpha}+\frac{2 C_{1} \Gamma(1+\alpha)\left(I_{n}+C_{1}^{2}(\Gamma(1+\alpha))^{2}\right)}{\Gamma(1+3 \alpha)} t^{3 \alpha}+\cdots .
$$

Note that so many new special cases can be extracted by giving two constant matrices $C_{0}$ and $C_{1}$.

\section{Conclusions}

In this work, we extend the FPS to the MFPS and discuss the convergence and radii of convergence for MFPS in Caputo sense. In addition, we approximate the matrix fractional derivatives and fractional integrals of a given matrix function with respect to Frobenius norm. Finally, new techniques are also applied for getting the general exact (numerical) solutions of some linear and non-linear MFDEs. How to apply our new method for solving systems of MFDEs such as matrix fractional time-varying descriptor system and matrix fractional control systems still need further researches. 


\section{Acknowledgment}

The author would to express his appreciation and thanks to Al-Balqa Applied University for granting him as a sabbatical leave which made this research possible.

\section{References}

[1] Z. Al-Zhour, Efficient solutions of coupled matrix and matrix differential equations, Intell. Control Auto., 3 (2012), 12 pages. 1,1

[2] Z. A. A. Al-Zhour, The general (vector) solutions of such linear (coupled) matrix fractional differential equations by using Kronecker structures, Appl. Math. Comput., 232 (2014), 498-510. 1, 1

[3] Z. A. A. Al-Zhour, New techniques for solving some matrix and matrix differential equations, Ain Shams Eng. J., 6 (2015), 347-354. 1

[4] Z. Al-Zhour, The general solutions of singular and non-singular matrix fractional time-varying descriptor systems with constant coefficient matrices in Caputo sense, Alexandaria Eng. J., 55 (2016), 1675-1681. 1, 1, 1

[5] Z. Al-Zuhiri, Z. Al-Zhour, K. Jaber, The exact solutions of such coupled linear matrix fractional differential equations of diagonal unknown matrices by using Hadamard product, J. Appl. Math. Phys., 4 (2016), 432-442. 1

[6] A. Atangana, J. J. Nieto, Numerical solution for the model of RLC circuit via the fractional derivative without singular kernel, Adv. Mech. Eng., 7 (2015), 7 pages. 1

[7] K. Balachandran, J. Kokila, On the controllability of fractional dynamic systems, Int. J. Appl. Math. Comput. Sci., 22 (2012), 523-531. 1

[8] K. Balachandran, J. Kokila, J. J. Trujillo, Relative controllability of fractional dynamic systems with multiple delays in control, Comput. Math. Appl., 64 (2012), 3037-3045. 1

[9] A. Carpinteri, F. Mainardi, Fractional calculus: Some basic problems in continuum and statistical mechanics, in: Fractals and Fractional Calculus in Continuum Mechanics, 1997 (1997), 291-348. 1

[10] J. B. Cruz, C. I. Chen, Series Nash solution of two-person nonzero sum linear differential games, J. Optim. Theory Appl., 7 (1971), 240-257. 1

[11] M. Dalir, M. Bashour, Applications of fractional calculus, Appl. Math. Sci. (Ruse), 4 (2010), 1021-1032. 1

[12] S. Das, Functional Fractional Calculus for System Identification and Controls, Springer, Berlin, (2008).

[13] W. H. Deng, C. P. Li, Chaos Synchronization of the fractional Lu system, Phys. A, 353 (2005), 61-72. 1

[14] A. El-Ajou, O. Abu Arqub, M. Al-Smadi, A general form of the generalized Taylor's formula with some applications, Appl. Math. Comput., 256 (2015), 851-859. 1, 2, 2

[15] A. El-Ajou, O. Abu Arqub, S. Momani, Solving fractional two-point boundary value problems using continuous analytic method, Ain Shams Eng. J., 4 (2013), 539-547. 1

[16] A. El-Ajou, Z. Al-Zhour, M. Oqielat, S. Momani, T. Hayat, Series Solutions of Nonlinear Conformable Fractional KdV-Burgers Equation with Some Applications, Eur. Phys. J. Plus, 134 (2019), 11 pages. 1, 2

[17] A. El-Ajou, M. N. Oqielat, Z. Al-Zhour, S. Kumar, S. Momani, Solitary solutions for time-fractional nonlinear dispersive PDEs in the sense of conformable fractional derivative, Chaos, 29 (2019), 12 pages. 1

[18] A. El-Ajou, M. N. Oqielat, Z. Al-Zhour, S. Momani, Analytical numerical solutions of the fractional multi-pantograph system: Two attractive methods and comparisons, Results Phys., 14 (2019), 10 pages. 1, 2

[19] A. Graham, Kronecker Products and Matrix Calculus with Applications, Ellis Horwood Ltd., Chichester, (1981). 1, 1

[20] I. Grigorenko, E. Grigorenko, Chaotic dynamics of the fractional Lorenz system, Phys. Rev. Letter., 91 (2003), 12 pages. 1

[21] S. Hasan, A. El-Ajou, S. Hadid, M. Al-Smadi, S. Momani, Atangana-Baleanu fractional framework of reproducing kernel technique in solving fractional population dynamics system, Chaos Solitons Fractals, 133 (2020), 13 pages. 1

[22] O. Heaviside, Electromagnetic theory, Chelsea, New York, (1971). 1

[23] R. A. Horn, C. R. Johnson, Topics in Matrix Analysis, Cambridge University Press, Cambridge, (1991). 1

[24] L. Jódar, H. Abou-Kandil, Kronecker products and coupled matrix Riccati differential systems, Linear Algebra Appl., 121 (1989), 39-51. 1, 1

[25] A. Kadem, D. Baleanu, Fractional radiative transfer equation within Chebyshev spectral approach, Comput. Math. Appl., 59 (2010), 1865-1873. 1

[26] A. Kilicman, W. A. Ahmood, On matrix fractional differential equations, Adv. Mech. Eng., 9 (2017), 7 pages. 1

[27] A. Kilicman, Z. A. A. Al-Zhour, Kronecker operational matrices for fractional calculus and some applications, Appl. Math. Comput., 187 (2007), 250-265. 1

[28] J. G. Lu, G. R. Chen, A note on the fractional-order Chen system, Chaos Solitons Fractals, 27 (2006), 685-688. 1

[29] R. L. Magin, Fractional calculus in bioengineering, Crit. Rev. Biomed. Eng., 32 (2004), 1-104. 1

[30] J. R. Magnus, H. Neudecker, Matrix Differential Calculus with Applications in Statistics and Econometrics, John Wiley \& Sons, Chichester, (1999). 1, 1

[31] K. S. Miller, B. Ross, An Introduction to the fractional calculus and fractional differential equations, John Wiley \& Sons, New York, (1993). 1 
[32] Z. M. Odibat, Analytic study on linear systems of fractional differential equations, Comput. Math. Appl., 59 (2010), 1171-1183. 1

[33] Z. M. Odibat, N. T. Shawagfeh, Generalized Taylor's formula, Appl. Math. Comput., 186 (2007), 286-293. 1

[34] K. B. Oldham, J. Spanier, The Fractional Calculus, Academic Press, New York, (1974). 1

[35] I. Podlubny, Fractional Differential Equations, Academic Press, San Diego, (1999). 1

[36] C. Riccardo, D. Giovanni, F. Luigi, Fractional Order System: Modeling and Control Applications, World Scientific, London, (2010).

[37] B. Ross, Fractional Calculus and its Applications, Springer-Verlag, Berlin, (1975). 1

[38] Y. Rossikhin, M. Shitikova, Applications of fractional calculus to dynamic problems of linear and nonlinear hereditary mechanics of solids, Appl. Mech. Rev., 50 (1997), 15-67. 1

[39] M. Shqair, A. El-Ajou, M. Nairat, Analytical Solution for Multi-Energy Groups of Neutron Diffusion Equations by a Residual Power Series Methods, Mathematics, 7 (2019), 20 pages. 1

[40] J. Wang, Y. Zhang, Network synchronization in a population of star-coupled fractional nonlinear oscillators, Phys. Lett. A, 374 (2010), 1464-1468. 1 